\title{
Spinal Neurocysticercosis Manifesting as Recurrent Aseptic Meningitis
}

\author{
Mark I. Boulos, Richard I. Aviv, Liesly Lee
}

Can. J. Neurol. Sci. 2010; 37: 878-880

Neurocysticercosis (NCC), the infection of the human brain by the larvae of the parasite Taenia solium, is the most common parasitic infection of the nervous system ${ }^{1}$. In countries where the parasite is endemic, NCC is a common reason for hospital admissions and a major cause of acquired epilepsy ${ }^{2}$. Neurocysticercosis is being diagnosed more frequently in industrialized countries because of immigration from endemic zones and tourism in these regions ${ }^{3}$.

Most NCC cysts are located in the subarachnoid spaces, typically the basal cisterns and deep within the sulci ${ }^{4}$. Other common locations include the hemispheric parenchyma at the gray-white matter junctions and the fourth ventricle; rarely, cysts are found around the spinal cord ${ }^{4}$, or near the cervicomedullary junction $^{5}$.

We report an unusual manifestation of NCC in a Canadian female who presented with recurrent bouts of debilitating headaches and aseptic meningitis. Initial magnetic resonance imaging (MRI) demonstrated subtle intracranial leptomeningeal enhancement, but this finding was only appreciated months after the original imaging. Her diagnosis remained unknown until subsequent imaging revealed a mass in the cervicomedullary junction, and the lesion was biopsied. Despite an incomplete surgical resection, she responded well to anthelmintic and steroid therapy.

\section{Report Of CASE}

The patient was a 35-year-old female law clerk who presented to our clinic in 2006. She had immigrated in 1973 from El Salvador to Canada at the age of two years, and had travelled back to her home country in 2001 for a brief holiday. She had no chronic illnesses aside from iron deficiency for which she had been using iron supplements.

Her first neurological symptoms began in 1999 when she presented with headaches and was diagnosed with aseptic meningitis following a lumbar puncture. She recovered fully and remained well but then developed recurrent non-specific but non-debilitating headaches in 2003.

In December 2005, she developed a severe headache that had developed gradually and persisted for two weeks. She denied other neurological or constitutional symptoms. Her vital signs and systemic examination were unremarkable. On neurological examination, fundoscopy revealed blurred disc margins bilaterally. Magnetic resonance imaging of the brain with gadolinium was initially reported as normal. Several months later, when subsequent imaging took place, the initial imaging was reviewed by a second neuroradiologist (RIA), who identified the presence of leptomeningeal enhancement over the anterior pontine surface and the parieto-occipital sulci. Spinal fluid examination revealed an elevated white cell count $(116 \mathrm{x}$ $\left.10^{6} / \mathrm{L}\right)$ with a lymphocytic predominance (102 x 106/L), high protein $(683 \mathrm{mg} / \mathrm{L})$ and low glucose $(0.2 \mathrm{mmol} / \mathrm{L})$. Cerebrospinal fluid (CSF) bacterial, viral, fungal and acid fast bacillus cultures were negative. The opening CSF pressure was not elevated. Routine bloodwork was within normal limits. A provisional diagnosis of recurrent aseptic meningitis was made, and amitriptyline and gabapentin were used in attempt to manage her headaches. Two months after admission, her disc margins were no longer blurred.

However, over the ensuing ten months, she required admission on two occasions for recurrent bouts of severe refractory headaches, episodic left facial paraesthesias, and one episode of right facial weakness. Repeat lumbar punctures demonstrated a similar profile with no evidence of malignant cells or infective cultures. Repeat MRI of the brain with gadolinium was unchanged. On the second admission, however, in October 2006, computed tomography and MRI identified a small $(1.6 \mathrm{~cm} \times 0.6 \mathrm{~cm})$ homogeneously-enhancing soft tissue mass posterior to the cervicomedullary junction in association with leptomeningeal enhancement (Figure A \& B). Mild ventricular enlargement, in comparison to prior studies, was also noted.

The patient subsequently underwent a posterior fossa craniectomy, C1 laminectomy and partial excision of the intradural mass. Intra-operatively, the lesion appeared to be a collapsed cyst and consisted of a white avascular membrane. In addition, there was a pink, vascular-appearing lesion that was adherent to both the vertebral artery and arachnoid. The cystic portion was excised and sent to pathology for analysis. Small biopsies were taken from the vascular-appearing lesion, but total excision was not attempted because of the lesion's proximity to the vertebral artery.

The final surgical neuropathology report described remnants of cysticerci (Figure C). A serum immunoblot assay conducted by the Canadian National Reference Centre for Parasitology also confirmed the diagnosis of cysticercosis. Computed tomography scan of the thighs to screen for dissemination of the parasite was unremarkable.

\footnotetext{
From the Department of Medicine (Neurology) (MIB, LL), Department of Neuroradiology (RIA), Sunnybrook Health Sciences Centre, Toronto, Ontario, Canada. Received December 23, 2009. Final Revisions Submitted June 22, 2010. Correspondence to: Liesly Lee, Sunnybrook Health Sciences Centre, A411 - 2075 Bayview Avenue, Toronto, Ontario, M4N 3M5, Canada.
} 


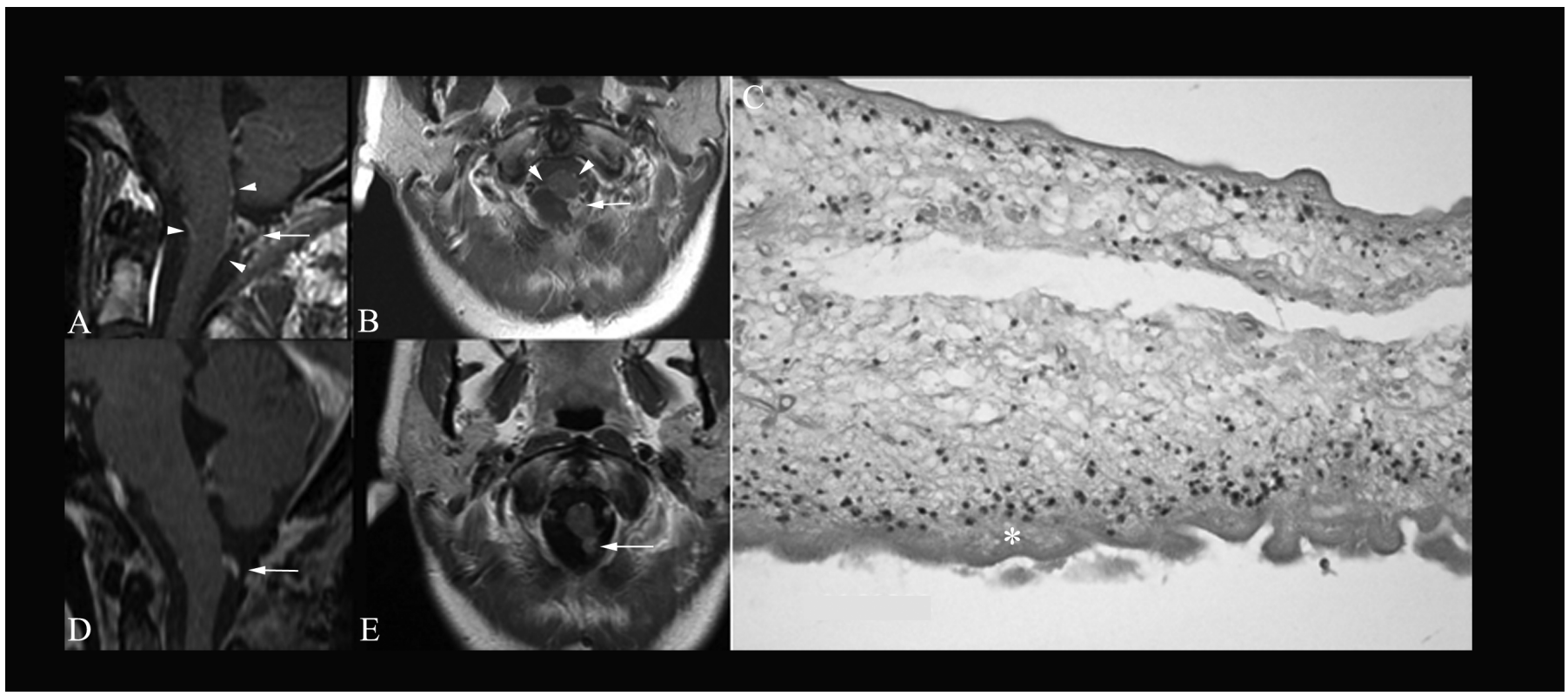

Figure: (A) Sagittal and (B) axial T1 post-gadolinium imaging demonstrated a nodule of enhancement posterolateral to the cervicomedullary junction (arrows). Leptomeningeal enhancement was seen along the cervicomedullary junction and adjacent nerve roots (arrowheads). (C) Neuropathology specimen demonstrated the cysticercus wall with microvilli on the tegument surface, a layer of tegument cells and, beneath, a layer of tegument cells and further a loose stroma with excretory holes. The asterisk marks the tegument. ( $D \& E)$ Follow-up contrast enhanced T1 studies demonstrated a reduction in the size of the enhancing nodule (arrows) and near-complete resolution of the leptomeningeal enhancement. Post-operative changes are seen within the posterior suboccipital soft tissue consistent with surgical biopsy.

The patient received two 2-week courses of Albendazole 400 mg twice daily that were separated by a month, along with Prednisone $30 \mathrm{mg}$ daily, which was subsequently tapered. Repeat MRI, conducted one year after surgery, demonstrated a reduction in the size of the lesion and reduced pial enhancement (Figure D $\&$ E). The patient's headaches resolved and she returned to her previous high functioning level.

\section{Discussion}

Our case demonstrates biopsy-confirmed NCC in an unusual location: the cervicomedullary junction in an intradural, extramedullary region. Most series report the incidence of spinal NCC to be only 1.5 to $3 \%$ of all cases ${ }^{6}$. As in our patient, intracranial involvement is usually present in spinal NCC, with isolated spinal forms being rare $^{7}$. Intradural spinal manifestations can be divided into extramedullary (subarachnoid) and intramedullary forms, the former being more common ${ }^{8}$. Brainstem involvement has also been reported in NCC, often in association with midbrain or pontine lesions ${ }^{9,10}$; in contrast, far fewer medullary ${ }^{11}$ or cervicomedullary ${ }^{5}$ lesions are discussed in the literature.

The treatment of NCC is controversial, and remains based primarily on poorly-controlled studies or anecdotal evidence ${ }^{12}$. Extraparenchymal manifestations, such as those in the subarachnoid spaces, ventricles or around the spinal cord, are particularly difficult to manage as the prognosis of these patients is worse compared with those having only parenchymal disease $^{13}$. Extraparenchymal lesions may or may not improve with anthelmintic treatment and, in contrast to parenchymal cysts, often need multiple courses of anthelmintics and the use of concomitant steroids; surgery may be required in life-threatening cases $^{14}$. Complications associated with this form of NCC include meningeal inflammation, which can cause chronic meningitis and hydrocephalus; cranial nerve findings can occur as a result of granulomatous inflammation affecting nearby nerves ${ }^{12}$, as likely occurred in our patient.

Because of its rarity, the management of spinal NCC is poorly defined, but surgical intervention is commonly recommended ${ }^{2,3}$. In non-acute intradural intramedullary spinal cysticercosis, however, several case reports describe successful outcomes with the use of anthelmintic agents alone, and emphasize the need for accurate pre-operative imaging to avoid surgery in selected cases $^{15}$. In extramedullary (subarachnoid) spinal NCC, most authors describe combined medical and surgical approaches ${ }^{6,8}$. With the current case, we also utilized a combined management regime. Our report demonstrates that, with appropriate medical therapy, a successful outcome can take place in extramedullary spinal NCC despite an incomplete surgical excision of the lesion.

An unusual feature of the present case was the lengthy period between exposure and disease manifestation. While the natural history of human NCC is poorly understood, natural epidemiological observations have shown that most patients develop symptoms within two to five years after exposure ${ }^{3}$. The patient was only two-years-old when she left her home country of El Salvador in 1973; her initial symptoms and the concomitant diagnosis of aseptic meningitis manifested in 1999, about 26 years later. Her symptoms had already started when she returned to visit her home country in 2001 . Our case demonstrates the 
potentially lengthy incubation period of NCC. However, the possibility of autoinfection should also be considered, as the adult tapeworm can remain in the intestine for many years or decades, and at any time autoinfection can occur through reverse peristalsis, with subsequent hatching of the parasite's eggs in the stomach $^{16}$. Alternatively, person-to-person transmission could have occurred any time after the patient's arrival in Canada, provided there was a symptom-free tapeworm carrier in her household ${ }^{3}$.

The clinical presentation of NCC depends on the number, size and locations of the cysticercal cysts within the central nervous system and the host's immune response to the parasite ${ }^{16}$. Our patient's recurrent bouts of aseptic meningitis and refractory headaches resulted from the local invasion of NCC in the cervicomedullary junction and the concurrent subarachnoid spread to the brainstem and parieto-occipital cortices; this also gave rise to the leptomeningeal involvement observed on imaging, as well as the facial sensory and motor symptoms. Her blurred disc margins were likely secondary to leptomeningeal seeding and the elevated CSF white cell count. We postulate that a cysticercal cyst ruptured prior to the discovery of a mass on imaging and her clinical worsening. Her enlarged ventricles presumably resulted from mass effect of the ruptured cyst on the central canal, as well as the secondary inflammatory response.

Our case demonstrates that the initial presentation of NCC can be elusive, with only subtle leptomeningeal enhancement seen in association with severe headaches and recurrent aseptic meningitis. We show that NCC may have an incubation period of several decades, that the parasitic infection can arise in extraaxial locations other than those typically reported, and that subarachnoid spinal lesions can have favourable outcomes with anthelmintic and steroid therapy despite an incomplete surgical resection. Despite a patient's low pre-test probability of having NCC, given its increasing prevalence in developed countries via immigration and tourism, NCC should be regularly considered in the differential diagnosis of recurrent aseptic meningitis.

\section{ACKNOWLEDGEMENTS}

The authors thank Dr. J. Bilbao for his description of the neuropathology. Informed consent was obtained for publication of the patient's details in this report.

\section{REFERENCES}

1. Del Brutto OH. Neurocysticercosis. Semin Neurol. 2005;25: 243-51.

2. Garcia HH, Gonzalez AE, Evans CAW, Gilman RH, for the Cysticercosis Working Group in Peru. Taenia solium cysticercosis. Lancet. 2003;362:547-56.

3. Garcia HH, Del Brutto OH, for the Cysticercosis Working Group in Peru. Neurocysticercosis: updated concepts about an old disease. Lancet Neurol. 2005;4:653-61.

4. Osborn AG, Preece MT. Intracranial cysts: radiologic-pathologic correlation and imaging approach. Radiology. 2006;239:650-64.

5. Davies MA, Turner J, Bentivoglio P. Spinal and basilar extraparenchymal neurocysticercosis. J Clin Neurosci. 1996;3 (2):174-7.

6. Alsina GA, Johnson JP, McBride DQ, Rhoten PR, Mehringer CM, Stokes JK. Spinal neurocysticercosis. Neurosurg Focus. 2002; 12:e8.

7. Leite CC, Jinkins JR, Escobar BE, Magalhaes AC, Gomes GC, Dib $\mathrm{G}$, et al. MR imaging of intramedullary and intraduralextramedullary spinal cysticercosis. AJR Am J Roentgenol. 1997;169:1713-17.

8. Paterakis KN, Kapsalaki E, Hadjigeorgiou GM, Barbanis S, Fezoulidis I, Kourtopoulos H. Primary spinal intradural extramedullary cysticercosis. Surg Neurol. 2007;68:309-12.

9. Patel R, Jha S, Yadav RK. Pleomorphism of the clinical manifestations of neurocysticercosis. Am J Trop Med Hyg. 2006; 100:134-41.

10. Bhatia R, Desai S, Garg A, Padma MV, Prasad K, Tripathi M. Isolated facial myokymia as a presenting feature of pontine neurocysticercosis. Mov Disord. 2008;23:135-7.

11. Garg RK. Wallenberg's lateral medullary syndrome: a new nonvascular cause. J Assoc Physicians India. 1999;47:1110-11.

12. Nash TE, Singh G, White AC, Rajshekhar V, Loeb JA, Proaño JV, et al. Treatment of neurocysticercosis: current status and future research needs. Neurology. 2006;67:1120-7.

13. Estanol B, Corona T, Abad P. A prognostic classification of cerebral cysticercosis: therapeutic implications. J Neurol Neurosurg Psychiatry. 1986;49:1131-4.

14. Proaño JV, Madrazo I, Avelar F, López-Félix B, Díaz G, Grijalva I. Medical treatment for neurocysticercosis characterized by giant subarachnoid cysts. N Engl J Med. 2001;345:879-85.

15. Ahmad FU, Sharma BS. Treatment of intramedullary spinal cysticercosis: report of 2 cases and review of literature. Surg Neurol. 2007;67:74-7.

16. Hawk MW, Shahlaie K, Kim KD, Theis JH. Neurocysticercosis: a review. Surg Neurol. 2005;63:123-32. 